\title{
latrogenic Exserohilum infection of the central nervous system: mycological identification and histopathological findings
}

W Robert Bell ${ }^{1,5}$, Justin B Dalton ${ }^{1,5}$, Chad M McCall ${ }^{1,5}$, Sarah Karram ${ }^{1,5}$, David T Pearce ${ }^{1}$, Warda Memon ${ }^{1}$, Richard Lee ${ }^{1}$, Karen C Carroll ${ }^{1}$, Jennifer L Lyons ${ }^{2}$, Elakkat D Gireesh ${ }^{2}$, Julie B Trivedi ${ }^{3}$, Deanna Cettomai ${ }^{2}$, Bryan R Smith ${ }^{2}$, Tiffany Chang ${ }^{4}$, Laura Tochen ${ }^{2}$, John N Ratchford ${ }^{2}$, Daniel M Harrison ${ }^{2}$, Lyle W Ostrow ${ }^{2}$, Robert D Stevens ${ }^{2,4}$, Li Chen ${ }^{1}$ and Sean X Zhang ${ }^{1}$

${ }^{1}$ Department of Pathology, Johns Hopkins University School of Medicine, Baltimore, MD, USA;

${ }^{2}$ Department of Neurology, Johns Hopkins University School of Medicine, Baltimore, MD, USA;

${ }^{3}$ Department of Medicine, Division of Infectious Diseases, Johns Hopkins University School of Medicine, Baltimore, MD, USA and ${ }^{4}$ Department of Anesthesiology Critical Care Medicine, Johns Hopkins School of Medicine, Baltimore, MD, USA

An outbreak of fungal infections has been identified in patients who received epidural injections of methylprednisolone acetate that was contaminated with environmental molds. In this report, we present the mycological and histopathological findings in an index case of Exserohilum meningitis and vasculitis in an immunocompetent patient, who received a cervical spine epidural steroid injection for chronic neck pain 1 week before the onset of fulminant meningitis with subsequent multiple brain and spinal cord infarcts. The fungus was recovered from two separate cerebrospinal fluid specimens collected before initiation of antifungal therapy and at autopsy on standard bacterial and fungal culture media. The mold was identified phenotypically as Exserohilum species. DNA sequencing targeting the internal transcribed spacer region and D1/D2 region of 28S ribosomal DNA enabled further speciation as E. rostratum. Gross examination at autopsy revealed moderate brain edema with bilateral uncal herniation and a ventriculostomy tract to the third ventricle. The brainstem, cerebellum, and right orbitofrontal cortex were soft and friable, along with hemorrhages in the cerebellar vermis and thalamus. Microscopic examination demonstrated numerous fungi with septate hyphae invading blood vessel walls and inducing acute necrotizing inflammation. The leptomeninges were diffusely infiltrated by mixed inflammatory cells along with scattered foci of fungal elements. This is the first report of iatrogenic $E$. rostratum meningitis in humans. This report describes the microbiological procedures and histopathological features for the identification of $E$. rostratum (a pigmented vascularly invasive fungi), the cause of a current nationwide outbreak of fatal fungal meningitis.

Modern Pathology (2013) 26, 166-170; doi:10.1038/modpathol.2012.208; published online 7 December 2012

Keywords: epidural steroids; Exserohilum rostratum; Fungal meningitis; methylprednisolone; outbreak; vasculitis

Dematiaceous fungi are ubiquitous in the environment. They are characterized by the presence of

Correspondence: Dr L Chen, MD, PhD, Division of Neuropathology, Department of Pathology Johns Hopkins University School of Medicine, 720 Rutland Avenue - Ross Building 519, Baltimore, MD 21205, USA.

E-mail: lchen99@jhu.edu or Dr S Zhang, MD, PhD, Division of Medical Microbiology, Department of Pathology, Johns Hopkins University School of Medicine, The Johns Hopkins Hospital, 600 North Wolfe Street, Meyer B1-193, Baltimore, MD 21287-7093, USA. E-mail: szhang28@jhmi.edu

${ }^{5}$ These authors contributed equally to this work.

Received 26 October 2012; revised 2 November 2012; accepted 2 November 2012; published online 7 December 2012 melanin in their cell walls and are not a common cause of human disease. Nonetheless, a spectrum of clinical presentations associated with dematiaceous fungi has been reported including superficial and deep local infections, allergic disease, pneumonia, brain abscess and disseminated disease. ${ }^{1}$ Infection is thought to result from either trauma to the skin, inhalation, or hematogenous spread from a remote sub-acute infection. ${ }^{1,2}$ In the central nervous system, the formation of a brain abscess has been the most common finding, frequently occurring in immunocompetent individuals. ${ }^{2}$ In 2002, iatrogenic meningitis caused by a dematiaceous fungus Exophiala was observed as a complication of contaminated 
epidural glucocorticoid injections. ${ }^{3,4}$ Presently, the Exserohilum has been identified as the most common and important organism in an ongoing multistate outbreak after an initial alert was generated due to an Aspergillus infection.,6 Given the rarity of these organisms as causative agents of meningitis, accurate diagnosis rests largely on mycological and pathologic description. Here, we present the mycological and histopathological findings in one of the index cases of Exserohilumassociated meningitis and vasculitis in the current nationwide outbreak, the clinical and neuroradiological findings of which were recently reported.

\section{Materials and methods}

Cerebrospinal fluid (CSF) specimens collected both preand post-mortem were inoculated into standard bacterial and fungal culture media: 5\% sheep blood, chocolate agar, Schaedler enrichment broth, Sabouraud dextrose agar, brain heart infusion agar with or without $10 \%$ sheep blood. A mold that grew on these media was sub-cultured to potato flake agar, a plant-based medium to support sporulation of the mold for identification. Lactophenol cotton blue preparations were used to visualize fungal organisms. Sequencing of D1/D2 of 28S rDNA and internal transcribed spacer regions was performed using an ABI Prism 3100 DNA Analyzer (Applied Biosystems, Foster City, CA, USA). Antifungal susceptibility testing was performed at the Fungus Testing Laboratory, Department of Pathology, University of Texas Health Sciences Center (San Antonio, TX, USA) using a broth microdilution method recommended by the Clinical and Laboratory Standards Institute. ${ }^{7}$

The autopsy was limited to brain and spinal cord, with appropriate consent from the patient's next of kin. The specimen was fixed in 10\% formalin for 3 weeks following the initial gross examination, after which serial coronal sections were made and multiple sections of the cerebral cortex, basal ganglia, thalamus, cerebellum, midbrain, pons, medulla, and spinal cord were obtained for microscopic examination using a hematoxylin and eosin (H\&E) stain. To further examine the presence and morphology of the fungal organism, selected sections were stained using Grocott's methenamine silver and Fontana-Masson stains.

\section{Results}

\section{Microbiological Findings}

Two CSF samples were collected from the patient: 1 day before initiation of antifungal therapy from the third ventricle, and 4 days later at autopsy from the thecal sac. The pre-mortem culture showed very light growth of a white, velvety filamentous organism 2 days after plating on 5\% sheep blood agar and chocolate agar and 3 days after inoculation in Schaedler enrichment broth; however, the fungus failed to grow on Sabouraud agar and brain heart infusion agar. A lactophenol cotton blue preparation of the filamentous organism showed hyaline and septate hyphae without sporulation. A second lactophenol cotton blue preparation from the colony that grew on the potato flake agar at 2 weeks showed pigmented, septate hyphae with pale brown, geniculate conidiophores with conidia cylindrical to ellipsoidal, multicellular ( $\geq 6$ distoseptate), and a protuberant hilum at the base suggestive of Exserohilum spp (Figure 1a). Internal transcribed spacer sequence analysis identified the organism as Exserohilum rostratum. The post-mortem culture demonstrated growth of a filamentous organism on Sabouraud agar 3 weeks after collection (Figure 1b), which was morphologically identical to the Exserohilum species isolated from the pre-mortem sample. Both internal transcribed spacer and D1/D2 sequence analysis confirmed it as E. rostratum. Antifungal susceptibility testing of the initial culture isolate showed minimum inhibitory concentrations (MICs) for amphotericin B $0.06 \mu \mathrm{g} / \mathrm{ml}$;
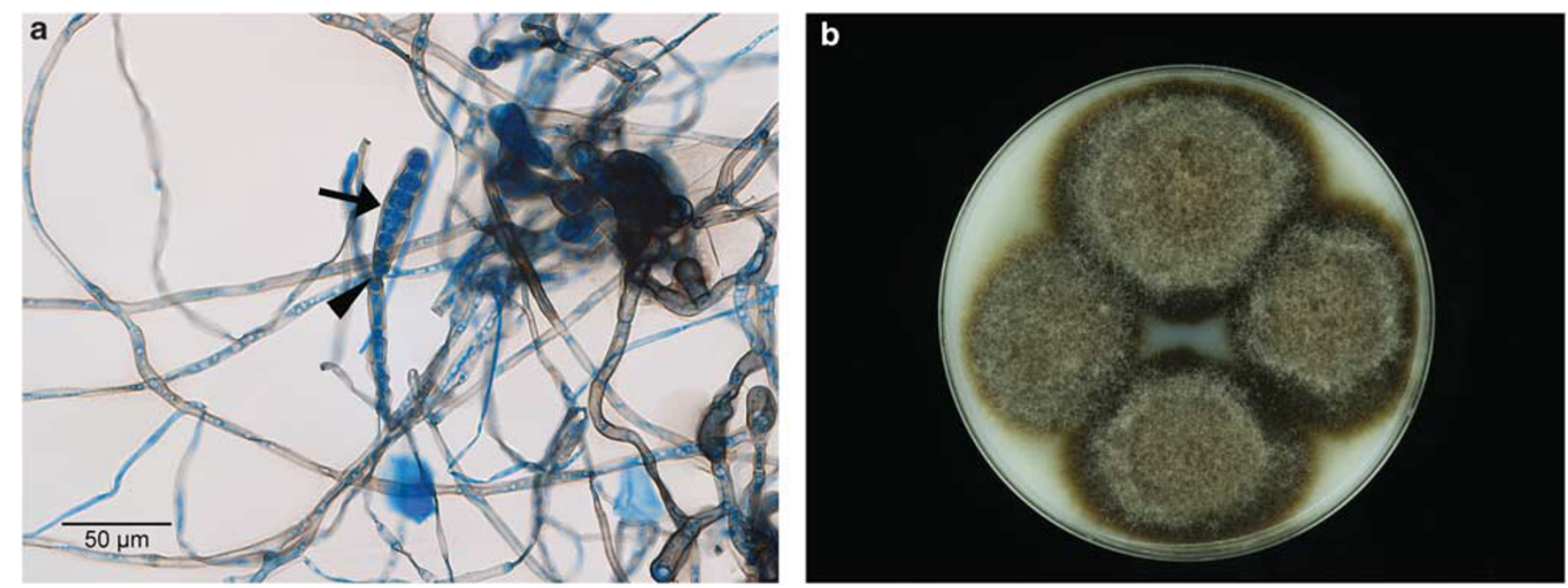

Figure 1 (a) Lactophenol cotton blue stain showed a conidium with $\geq 6$ distoseptate (arrow) and a hilum at the base (arrowhead). (b) Spreading, velvety to wooly, dark olive to black colonies on the surface of potato flake agar. 
micafungin $0.03 \mu \mathrm{g} / \mathrm{ml}$; fluconazole $32 \mu \mathrm{g} / \mathrm{ml}$; itraconazole $0.5 \mu \mathrm{g} / \mathrm{ml}$; and voriconazole $2 \mu \mathrm{g} / \mathrm{ml}$.

\section{Autopsy Gross Findings}

The fresh brain weight was $1180 \mathrm{~g}$. External examination revealed diffuse brain edema with bilateral uncal herniation and evidence of a ventriculostomy entry site on the surface of the right frontal lobe. The leptomeninges over the base of brain and cerebellum were thickened. Vertebrobasilar arteries and vessels of the circle of Willis were discolored and flaccid. The right orbitofrontal cortex, brainstem (in particular, the midbrain and pons), cerebellum (left more severe than right), and the cervical and upper thoracic spinal cord were softened and friable. Coronal sectioning traced the ventriculostomy tract to the third ventricle. Hemorrhages in the cerebellar vermis and left thalamus were identified.

\section{Histopathological Findings}

Microscopic examination further revealed a global hypoxic-ischemic injury to the brain and spinal cord. Acute and chronic inflammation with scattered foci of fungal elements was evident throughout the leptomeninges (Figure 2a). Necrotic vessels with thrombosis and hemorrhages were noted (Figure 2b), particularly in areas overlying the cervical and upper thoracic spinal cord. Representative sections of vertebrobasilar arteries and branches of the circle of Willis demonstrated numerous filamentous fungi extending the full thickness of the vessel wall, admixed with a largely neutrophillic infiltrate, destroying the internal elastic lamina. The long and moderately broad hyphae $\sim 5-10 \mu \mathrm{m}$ in diameter were identified on H\&E staining and best visualized by special stains such as Grocott's methenamine silver (Figure 2c). The hyphae exhibited occasional branching and regular septation. A Fontana-Masson stain further confirmed the presence of melanin pigment in hyphae cell walls (Figure 2d). Parenchymal hemorrhages (Figure 2e) and areas of infarction (Figure 2f) were seen in the cerebellum, thalamus, and spinal cord. Widespread hypoxic neuronal changes (eosinophilic neurons) and neuropil vacuolation were observed, most prominently in the hippocampus and cerebellum.

\section{Discussion}

Dematiaceous fungi are ubiquitous in the environment and as such are sometimes encountered as laboratory contaminants. ${ }^{1}$ Without a strong clinical suspicion, such organisms may be overlooked as the cause of infection, as they only rarely cause human disease. This report comprises the first detailed mycological identification and characterization of E. rostratum meningitis due to epidural injection of contaminated methylprednisolone. The autopsy findings illustrate the pathology of the diffuse meningitis and highlight the transmural and angioinvasive character of this fungal organism. The necrotic fungal vasculitis and thrombosis is the most striking characteristic and was the proximate cause of the patient's death, leading to infarcts and hemorrhages in the spinal cord, brainstem, thalamus, and cerebellum.

The mycological identification and characterization of E. rostratum in this case demonstrates the difficulty associated with the early diagnosis of fungal meningitis. Our initial isolate from the CSF grew on a sheep blood agar plate and a chocolate agar plate at day 2 of culture, but it did not grow on selective fungal culture media. There is no clear explanation, but sampling may be a contributing factor because the second isolate did eventually grow after 3 weeks on fungal culture media. The presumptive identification of the initial isolate as Exserohilum spp. was made microscopically based on morphological features at 2 weeks, when the organism started to sporulate on potato flake agar and produce conidia with features of $\geq 6$ distoseptate and a protuberant hilum at the base suggestive of Exserohilum spp. This presumptive identification was not made at day 10 of culture, in which the young conidia only exhibited $<6$ distoseptate and no hilum at the base, indistinguishable from another dematiaceous fungus Bipolaris spp., which usually produces conidia containing 3-6 distoseptate and no hilum. Even after 2 weeks, when the conidia developed into the features suggestive of Exserohilum spp., the definitive identification to the species level as E. rostratum (conidia mostly with 7-9 distoseptate and darkly pigmented bands at the ends) could not be confidently made by microscopic features. However, early identification of this fungus as $E$. rostratum was achieved by applying fungal DNA sequencing at day 10 of culture when this organism just started to sporulate. Therefore, based on our experience in this case, DNA sequence analysis was able to identify this organism to the correct species level in several days as opposed to several weeks for complete morphological identification. The fungal DNA sequence method for rapid identification is important in controlling outbreak, and in assisting clinicians to initiate treatment and further to develop recommendations to manage patients infected by this organism. ${ }^{8}$ Furthermore, if empiric antifungals have been initiated, positive cultures may be delayed: the autopsy CSF culture took 3 weeks for growth to be detectable as opposed to the initial CSF culture collected before antifungal treatment, which took 2 days to become positive.

Although Clinical and Laboratory Standards Institute interpretive breakpoints have not been established for Exserohilum spp., da Cunha et al ${ }^{9}$ have recently tested 34 clinical isolates of E. rostratum. In comparison with their results for amphotericin B (MIC range $<0.03-0.125 \mu \mathrm{g} / \mathrm{ml}$ ), 

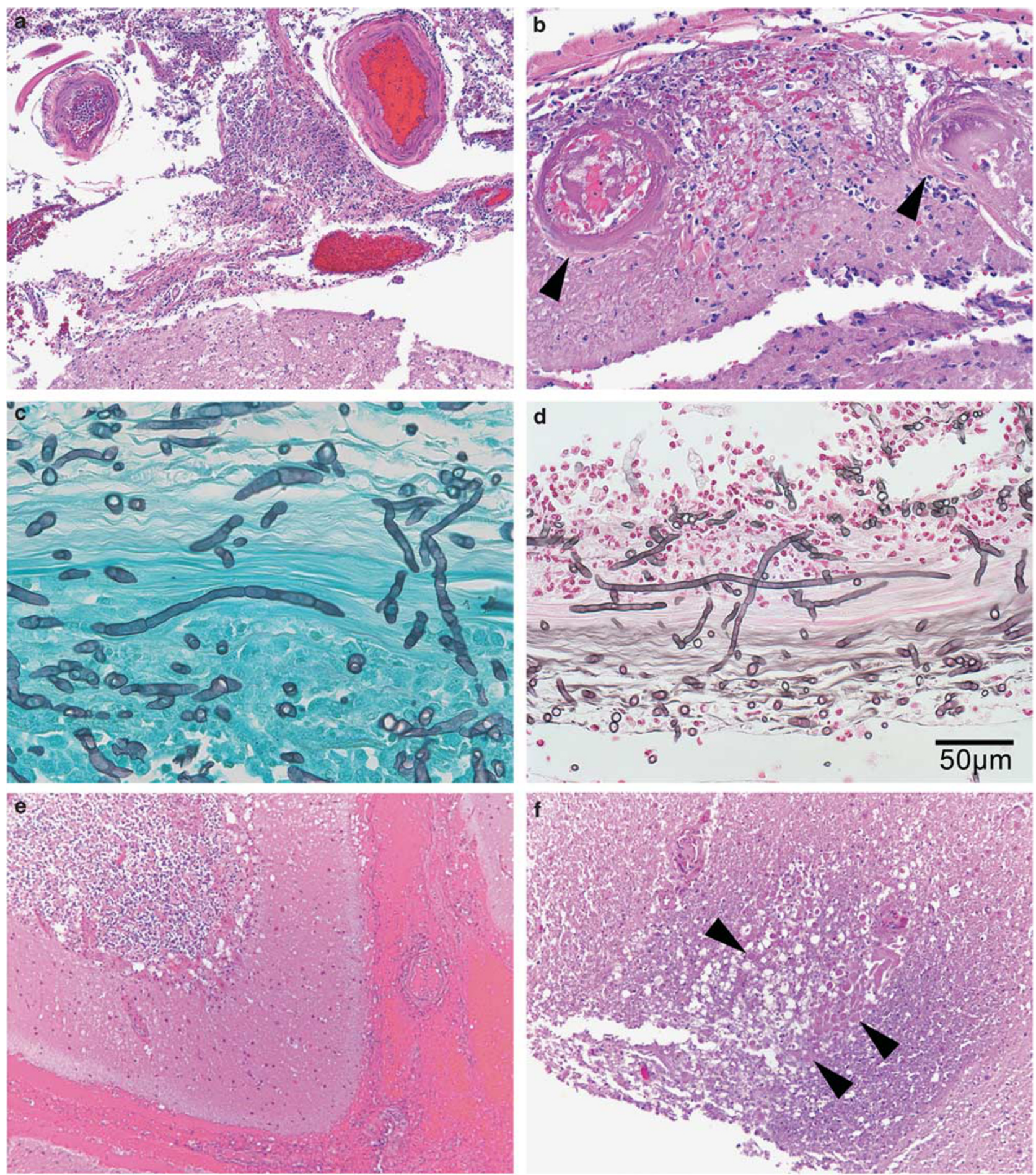

Figure 2 (a) Marked inflammation of the leptomeninges overlying the orbitofrontal cortex (H\&E stain, $\times 100$ magnification). (b) Thrombosed vessels with necrotic wall (arrowheads) in meninges covering the upper thoracic spinal cord. (H\&E stain, $\times 200$ magnification). (c) Broad septate hyphae with occasional branching invading the right middle cerebral artery. (Grocott's methenamine silver stain, $\times 260$ magnification, oil immersion). (d) Positive staining (brown to black) showing the presence of melanin pigment in the hyphae wall (Fontana-Masson stain, $\times 160$ magnification, oil immersion). (e) Hemorrhage and ischemic changes in the cerebellum. (H\&E stain, $\times 100$ magnification). (f) Spinal cord infarction (arrowheads highlight the axonal spheroids) (H\&E stain, $\times 200$ magnification).

itraconazole $\quad(<0.03-0.125 \mu \mathrm{g} / \mathrm{ml}), \quad$ voriconazole $(<0.03-1 \mu \mathrm{g} / \mathrm{ml})$, micafungin $(0.03$ to $>16 \mu \mathrm{g} / \mathrm{ml})$, the MICs for amphotericin B $(0.06 \mu \mathrm{g} / \mathrm{ml})$ and micafungin $(0.03 \mu \mathrm{g} / \mathrm{ml})$ of our isolate are similar.
However, the MICs for itraconazole $(0.5 \mu \mathrm{g} / \mathrm{ml})$ and voriconazole $(2 \mu \mathrm{g} / \mathrm{ml})$ for our isolate were elevated compared with their testing. At the present, voriconazole has been recommended as the drug of 
choice to treat the patients with fungal infections associated with the current outbreak. ${ }^{8}$ A limited number of isolates from this outbreak have been tested to date and their MICs of voriconazole ranged from 0.5 to $2 \mu \mathrm{g} / \mathrm{ml}^{8}{ }^{8}$ Whether elevated voriconazole MIC correlates with treatment failure is unknown at this point, but will be determined when more clinical information regarding the treatment of these infections becomes available.

This report illustrates both the extent and severity of neurologic disease possible in a patient with E. rostratum meningitis and vasculitis. Additionally, we provide a framework for identification and treatment of infections associated with the current outbreak of iatrogenic fungal meningitis.

\section{Disclosure/conflict of interest}

Sean X Zhang received contract work from IBIS/ Abbott Molecular. Karen C Carroll received research grants from Nanosphere, BioFire and BD Diagnostics, Scientific Advisory Boards: Quidel, NanoMR. The other authors declare no conflict of interest.

\section{References}

1 Revankar SG. Dematiaceous fungi. Mycoses 2007;50: 91-101.
2 Revankar SG, Sutton DA, Rinaldi MG. Primary central nervous system phaeohyphomycosis: a review of 101 cases. Clin Infect Dis 2004;38:206-216.

3 Centers for Disease Control and Prevention. Exophiala infection from contaminated injectable steroids prepared by a compounding pharmacyUnited States, July-November 2002. JAMA 2003;289: 291-293.

4 Exophiala infection from contaminated injectable steroids prepared by a compounding pharmacy-United States, July-November 2002. MMWR Morb Mortal Wkly Rep 2002;51:1109-1112.

5 Multistate outbreak of fungal Infection associated with injection of methylprednisolone acetate solution from a single compounding pharmacy - United States 2012) MMWR Morb Mortal Wkly Rep. 2012;61: 839-842.

6 Pettit AC, Kropski JA, Castilho JL, et al. The index case for the fungal meningitis outbreak in the United States. N Engl J Med 2012 (E-pub ahead of print).

7 Reference methods for broth dilution antifungal susceptibility testing of filamentous fungi: approved standard M38-A2, 2nd edn. Clinical Laboratory Standards Institute: Wayne, PA, 2008.

8 Kauffman CA, Pappas PG, Patterson TF. Fungal infections associated with contaminated methylprednisolone injections - preliminary report. N Engl J Med 2012 (E-pub ahead of print).

9 da Cunha KC, Sutton DA, Gene J, et al. Molecular identification and in vitro response to antifungal drugs of clinical isolates of Exserohilum. Antimicrob Agents Chemother 2012;56:4951-4954. 